\title{
Generalized Jacobi Weights, Christoffel Functions, and Zeros of Orthogonal Polynomials*
}

\author{
Tamás ERdélyt and Paul Nevai \\ Deparment of Mathematics, The Ohio State Ciniversity, \\ P. O. Box 3341, Columbus, Ohio 43210-0341 \\ Communicated by V. Totik
}

Received January 7, 1991

\begin{abstract}
We study generalized Jacobi weight functions in terms of their (generalized) degree. We obtain sharp lower and upper bounds for the corresponding Christoffe! functions, and for the distance of the consecutive zeros of the corresponding orthogonal polynomials. The novelty of our results is that our constants depend only on the degree of the weight function but not on the weight itself. $\bar{c} 1992$ Academic Press, Inc.
\end{abstract}

\section{INTRODUCTION AND NOTATION}

The sets of all real-valued algebraic polynomials and trigonometric polynomials of degree at most $n$ will be denoted by $\Pi_{n}$ and $H_{n}$, respectively. The function

$$
w(z)=|\omega| \prod_{i=1}^{k}\left|z-z_{j}\right|^{r_{1},} \quad \omega \neq 0, z_{j} \in \mathbb{C}, z \in \mathbb{C}, r_{j}>0
$$

will be called a generalized Jacobi weight function of degree

$$
N=\sum_{j=1}^{k} r_{j}
$$

with positive exponents. The set of all generalized Jacobi weight functions of degree at most $N$ with positive exponents will be denoted by $|\mathrm{GCAP}|_{N}$. This notation comes from the fact that the function $w$ defined by $(1.1)$ is

* This material is based upon work supported by the National Science Foundation under Grant DMS-8814488, and by Nato under Grant CRG.870806. 
the modulus of a generalized complex algebraic polynomial of degree $N$ defined by (1.2). In the trigonometric case, the function

$$
w^{\prime}(z)=|\omega| \prod_{j=1}^{k}\left|\sin \frac{z-z_{j}}{2}\right|^{r_{j}}, \quad \omega \neq 0, z_{j} \in \mathbb{C}, z \in \mathbb{C}, r_{j}>0,
$$

will be called a generalized trigonometric Jacobi weight function of degree

$$
N=\frac{1}{2} \sum_{j=1}^{k} r_{j}
$$

with positive exponents. The set of all generalized trigonometric Jacobi weight functions of degree at most $N$ with positive exponents will be denoted by $|\mathrm{GCTP}|_{N}$. The function $w$ defined by (1.3) is the modulus of a generalized complex trigonometric polynomial of degree $N$ defined by (1.4) which explains our notation.

Let $\alpha$ be a nonnegative, finite Borel measure in $[-1,1]$. Given $0<p<\infty$, we define the Christoffel function $\lambda_{n}(\alpha)$ for $n=1,2, \ldots$, by

$$
\lambda_{n}(\alpha, p, z)=\min _{Q \in \Pi_{n-1}} \int_{-1}^{1} \frac{|Q(t)|^{p}}{|Q(z)|^{p}} d \alpha(t), \quad z \in \mathbb{C}
$$

and the generalized Christoffel function $\lambda_{n}^{*}(\alpha)$ for real $n \geqslant 1$ by

$$
\lambda_{n}^{*}(\alpha, p, z)=\inf _{f \in|G C A P|} \int_{n-1}^{1} \frac{f^{p}(t)}{f^{p}(z)} d \alpha(t), \quad z \in \mathbb{C} .
$$

For $M>0$, we also introduce the functions

$$
\Delta_{M}(x)=\max \left\{M^{-1} \sqrt{1-x^{2}}, M^{-2}\right\}, \quad-1 \leqslant x \leqslant 1,
$$

and

$$
w_{M}(x)=\int_{|\varepsilon-x| \leqslant A_{m}(x)} w(t) d t, \quad-1 \leqslant x \leqslant 1,
$$

where we assume that $w(t)$ is defined if $|t-x| \leqslant \Delta_{M}(x)$.

For $g(\geqslant 0) \in L^{1}(0,2 \pi)$, the Szegö function $D(g)$ is defined by

$$
D(g, z)=\exp \left(\frac{1}{4 \pi} \int_{0}^{2 \pi} \log g(\theta) \frac{1+z e^{-i \theta}}{1-z e^{-i \theta}} d \theta\right), \quad|z|<1 .
$$

The boundary value $D\left(g, e^{i t}\right)$ can be defined as the non-tangential limit of $D(g, z)$; this exists for amost every real $t$. It is important to note that $D(g) \in H^{2}(|z| \leqslant 1)$, and $\left|D\left(g, e^{i t}\right)\right|^{2}=|g(t)|$ holds for almost every real $t$. Other properties of the Szegö function may be found in, e.g., [6, Chap. V]. 
In Sections 2 and 3, we give sharp lower and upper bounds for Christoffei functions and for generalized Christoffel functions in $[-1,1]$ corresponding to generalized Jacobi weight functions. These results are proved in Sections 6 and 7. In Section 4, we give sharp lower and upper bounds for the distance of consecutive zeros of orthogonal polynomials corresponding to generalized Jacobi weight functions with positive exponents. These results are proved in Section 8. The properties of generalized Jacobi weight functions with positive exponents were the subject of study in a series of recent papers [1-5]. A number of these results which are used in this paper are summarized in Section 5.

The novelty of the results in the present paper is that the constants depend only on the degree of the weight function but not on the weight itself. For fixed generalized Jacobi weights, all our principal results have already been known (sce [9, Thcorcm 6.3.28, p. 120, and Theorem 9.22, p. 166] for the results corresponding to Theorems $2.1,2.2,3.1$, and 4.1 , whereas the "fixed" version of Theorem 3.2 was essentially proved in $[7$. formula (13), p. 150]).

2. UPPER BOUNDS FOR $\lambda_{n}(\alpha, p, x)$ FOR $-1 \leqslant x \leqslant 1$

TheOREM 2.1. Given $0<p<\infty, 0 \leqslant \Gamma<\infty$, and $n=1,2, \ldots$, let $M=1+p(n-1) /(\Gamma+p+1)$. Then there exists an absolute constant $c_{1}>0$ such that

$$
\lambda_{n}(\alpha, p, x) \leqslant c_{1}^{\Gamma+p+1} w_{M}(x), \quad-1 \leqslant x \leqslant 1,
$$

for every measure $\alpha$ such that $d \alpha=w d t$ with a $w \in|G C A P|_{\Gamma}$.

THEOREM 2.2. Let $0<p<\infty, \quad 0 \leqslant \Gamma<\infty$. and $n=1,2, \ldots$. Let $w=w^{(T)} / w^{(B)}$, where $w^{(T)}$ and $w^{(B)}$ belong $10|\mathrm{GCAP}|_{\Gamma}$, and let $d x=w d t$. Let $d$ denote the number of different zeros of $w^{(B)}$. Then there exists an absolute constant $c_{2}>0$ such that

$$
\lambda_{n}(\alpha, p, x) \leqslant c_{2}^{\Gamma+p d+p+1_{1}{ }_{M}(x), \quad-1 \leqslant x \leqslant 1,}
$$

with

$$
M=\frac{p(n-1-d)-\Gamma}{2 \Gamma+4+p}
$$

whenever $M \geqslant 1$, and

$$
\lambda_{n}^{*}(\alpha, p, x) \leqslant c_{2}^{\Gamma+1} w_{M}(x), \quad-1 \leqslant x \leqslant 1,
$$


with

$$
M=\frac{p(n-1)-\Gamma}{2 \Gamma+4}
$$

whenever $M>0$.

3. LOWER BOUNDS FOR $\lambda_{n}^{*}(\alpha, p, x)$ FOR $-1 \leqslant x \leqslant 1$

Since $\lambda_{n}^{*}(\alpha, p) \leqslant \lambda_{n}(\alpha, p)$, we will give lower bounds for $\lambda_{n}^{*}(\alpha, p)$ instead of $\lambda_{n}(\alpha, p)$. Our first theorem deals with the case $d \alpha(t)=w(t) d t$, where $w \in|\mathrm{GCAP}|_{\Gamma}$, and the second one gives a lower bound in a more general case, when the weight function $w$ satisfies the Szegö condition of logarithmic integrability.

TheOREM 3.1. Given $0<p<\infty, \quad 0 \leqslant \Gamma<\infty$, and $1 \leqslant n<\infty$, let $M=1+p(n-1) /(\Gamma+p+1)$. Then there exists an absolute constant $c_{3}>0$ such that

$$
\lambda_{n}^{*}(\alpha, p, x) \geqslant c_{3}^{\Gamma+p+1} w_{M}(x), \quad-1 \leqslant x \leqslant 1,
$$

for every measure $\alpha$ such that $d \alpha=w d t$ with $w \in|\mathrm{GCAP}|_{r}$.

THEOREM 3.2. Let $w$ be a nonnegative, integrable weight function in $[-1,1]$ such that $\log (w(\cos \cdot)) \in I^{1}(-\pi, \pi)$. Let $0<p<\infty$, and let $d \alpha=w d t$. Then there exists an absolute constant $c_{4}>0$ such that

$$
\lambda_{n}^{*}(\alpha, p, x) \geqslant c_{4} \Delta_{p(n-1)+1}(x)\left|D\left(w(\cos \cdot), r e^{i \theta}\right)\right|^{2}, \quad-1 \leqslant x \leqslant 1,
$$

where $r=(p(n-1)+1) /(p(n-1)+3), x=\cos \theta$, and $D(g)$ is the Szegö function defined by (1.9).

\section{Zeros of Orthogonal Polynomials Corresponding to Generalized Jacobi Weights with Positive Exponents}

We will use the standard notations. Let $\alpha$ be a nonnegative, finite Borel measure with $\operatorname{supp}(\alpha) \subset[-1,1]$, and let $\left\{p_{n}(\alpha)\right\}_{n=0}^{\infty}$ denote the corresponding orthonormal polynomials. In addition, $\left\{x_{j, n}(\alpha)\right\}_{j=1}^{n}$ denote the zeros of $p_{n}(\alpha)$ in decreasing order, $x_{0, n}=1, x_{n+1, n}=-1$, and $x_{j, n}=\cos \theta_{j, n}$ for $j=1,2, \ldots, n$. The main goal of this paper is to establish sharp lower and upper bounds for the distance of consecutive zeros of orthogonal polynomials associated with generalized Jacobi weight functions with positive 
exponents. The novelty of these estimates lies in the fact that our constants depend only on the degree of the weight function.

THEOREM 4.1. Let $0 \leqslant \Gamma<\infty$ and let $d x=w d t_{\text {, where }} w \in|\mathrm{GCAP}|_{\Gamma}$. Then there exist two absolute constants $c_{5}>0$ and $c_{6}>0$ such that the zeros of the corresponding orthogonal polynomials satisfy

$$
\theta_{j, n}-\theta_{j-1, n} \leqslant \frac{c_{S}^{\Gamma+1}}{n}, \quad j=1,2, \ldots, n+1,
$$

and

$$
\theta_{j, n}-\theta_{j-1, n} \geqslant \frac{c_{6}^{\Gamma+1}}{n}, \quad j=2,3, \ldots, n
$$

for $n=1,2, \ldots$.

We believe that (4.2) actually holds for $j=1$ and $j=n+1$ as well, but, alas, our method does not seem to work in these cases. The latter would generalize [8, Theorem 3, p. 367; 9, Theorem 9.22, p. 166].

\section{Auxiliary Results}

The extremal properties of generalized Jacobi weight functions with positive exponents were studied in a number of recent papers. In this section we list those theorems which will be used to prove our new results in Sections 2-4. We will use a pointwise Remez-type inequality for which we introduce the class

$$
\begin{array}{r}
|\operatorname{GCAP}|_{N}(s)=\left\{f \in|\mathrm{GCAP}|_{N}: m(\{x \in[-1,1]: f(x) \leqslant 1\}) \geqslant 2-s\right\} \\
0<s<2
\end{array}
$$

where $m(A)$ denotes the Lebesgue measure of a measurable set $A \subset \mathbb{R}$. The following theorem was proved in [1, Theorem 4].

THEOREM 5.1. There exists an absolute constant $c_{7}>0$ such that, given $0 \leqslant N<\infty$ and $0<s \leqslant 1$, the inequality

$$
f(x) \leqslant \exp \left(c_{7} N \min \left\{\frac{s}{\sqrt{1-x^{2}}}, \sqrt{s}\right\}\right), \quad-1 \leqslant x \leqslant 1,
$$

holds for every $f \in|\operatorname{GCAP}|_{N}(s)$. 
We will need Nikol'skiî-type inequalities for the classes $|\mathrm{GCAP}|_{N}$ and $|\mathrm{GCTP}|_{N}$ as well (see Section 1 for the notations). For $0 \leqslant N<\infty$ and $f \in|\mathrm{GCAP}|_{N}$ or for $n=0,1,2, \ldots$, and $f \in I I_{n}$, let

$$
\|f\|_{\infty}=\max _{-1 \leqslant t \leqslant 1}|f(t)| \text { and }\|f\|_{p}=\left(\int_{-1}^{1}|f(t)|^{p} d t\right)^{1 / p}, \quad 0<p<\infty,
$$

whereas for $0 \leqslant N<\infty$ and $f \in|\mathrm{GCTP}|_{N}$ or for $n=0,1,2, \ldots$, and $f \in H_{n}$, let

$$
\|f\|_{\infty}=\max _{-\pi \leqslant \theta \leqslant \pi}|f(\theta)| \text { and }\|f\|_{p}=\left(\int_{-\pi}^{\pi}|f(\theta)|^{p} d \theta\right)^{1 / p}, \quad 0<p<\infty .
$$

The following two inequalities were proved in [5, Theorems 5 and 6$]$.

ThEOREM 5.2. Given $0 \leqslant N<\infty$, there exists an absolute constant $c_{8}>0$ such that

$$
\|f\|_{p} \leqslant\left[c_{8}(1+q N)\right]^{2 / q-2 / p}\|f\|_{q}, \quad 0<q<p \leqslant \infty,
$$

holds for cvery $f \in|\mathrm{GCAP}|_{N}$. For instance, $c_{8}=e^{2}(2 \pi)^{-1}$ is a suitable choice.

THEOREM 5.3. Given $0 \leqslant N<\infty$, there exists an absolute constant $c_{9}>0$ such that

$$
\|f\|_{p} \leqslant\left[c_{9}(1+q N)\right]^{1 / q-1 / p}\|f\|_{q}, \quad 0<q<p \leqslant \infty,
$$

holds for every $f \in|\mathrm{GCTP}|_{N}$. For instance, $c_{9}=e(4 \pi)^{-1}$ is a suitable choice.

If $f \in|\mathrm{GCAP}|_{N}$, where $0 \leqslant N<\infty$, then $g(\theta)=|f(\cos \theta) \sin \theta| \epsilon$ $|\mathrm{GCTP}|_{N+1}$, and, thus, applying Theorem 5.3 to the function $g$ with $q=1$ and $p=\infty$, we obtain

$$
f(x) \leqslant 2 c_{9} \frac{N+2}{\sqrt{1-x^{2}}} \int_{-1}^{1} f(t) d t, \quad-1 \leqslant x \leqslant 1,
$$

for every $f \in|\mathrm{GCAP}|_{N}$. This, together with Theorem 5.2, gives

THEOREM 5.4. Given $0 \leqslant N<\infty$, there exists an absolute constant $c_{10}>0$ such that

$$
f(x) \leqslant c_{10}\left(\Delta_{N+2}(x)\right)^{-1} \int_{-1}^{1} f(t) d t, \quad-1 \leqslant x \leqslant 1,
$$

holds for every $f \in|\mathrm{GCAP}|_{N}$. For instance, $c_{10}=e^{2}(2 \pi)^{-1}$ is a suitable choice. 
Using a linear transformation, we easily deduce from Theorem 5.2 that $w_{M}(x) \leqslant 2 A_{M}(x) \max _{|t-x| \leqslant \Delta_{M}(x)} w(t) \leqslant 2 c_{8}^{2}(\Gamma+1)^{2} w_{M}(x), \quad-1 \leqslant x \leqslant 1$,

holds for every $w \in|\mathrm{GCAP}|_{\Gamma}$, where $w_{M}$ is defined by (1.8).

To prove (4.2) in Theorem 4.1, we will need weighted Markov and Bernstein type inequalitics. The following results arc particular cases of $[4$, Theorems 1 and 3].

THEOREM 5.5. Given $n=0,1, \ldots$, and $0 \leqslant \Gamma<\infty$, there exists an absolute constant $c_{11}>0$ such that

$$
\left\|Q^{\prime} w_{\infty} \leqslant c_{11}(n+r)^{2}\right\| Q w^{\prime} \|_{\infty}
$$

holds for every algebraic polynomial $Q \in \Pi_{n}$ and weight $\mathrm{w} \in|\mathrm{GCAP}|_{\Gamma}$.

THEOREM 5.6. Given $n=0,1, \ldots$, and $0 \leqslant \Gamma<\infty$, there exists an absolute constant $c_{12}>0$ such that

$$
\sqrt{1-x^{2}}\left|Q^{\prime}(x) w(x)\right| \leqslant c_{12}(\Gamma+1)(n+\Gamma)\|Q w\|_{x}, \quad-1<x<1,
$$

holds for every $Q \in \Pi_{n}$ and $w \in|\mathrm{GCAP}|_{r}$.

With the notation of (1.7), and combining Theorems 5.5 and 5.6 , we obtain the inequality

$$
\Delta_{n}(x)\left|Q^{\prime}(x) w(x)\right| \leqslant c_{13}(\Gamma+1)^{2}\left\|Q_{w}\right\|_{\infty}, \quad-1 \leqslant x \leqslant 1,
$$

for every $Q \in \Pi_{n}$ and $w \in|\mathrm{GCAP}|_{\Gamma}$, where $n=1,2, \ldots, 0 \leqslant \Gamma<\infty$, and $c_{13}>0$ is an absolute constant.

We think that the factor $(\Gamma+1)$ in Theorems 5.6 can perhaps be dropped.

\section{ProOF OF TheOREMS 2.1 AND 2.2}

To prove Theorem 2.1 we need the following

Lemma 6.1. Given $a>0, b \in \mathbb{R}$, and $0 \leqslant \Gamma<\infty$, the inequality

$$
w(y) \leqslant 2^{\Gamma} a^{-\Gamma}|y-b|^{\Gamma} \max _{b-a \leqslant t \leqslant b+a} w(t), \quad y \in \mathbb{R} \backslash(b-a, b+a),
$$

holds for every $w \in|\mathrm{GCAP}|_{\Gamma}$. 
Proof of Lemma 6.1. Without loss of generality we may assume that $a=1$ and $b=0$; the general case follows from this case by a linear substitution. Let $T_{m}$ denote the Chebyshev polynomial of degree $m$, and let $-1<x_{1, m}<x_{2, m}<\cdots<x_{m, m}<1$ denote the zeros of $T_{m}$, where $x_{j, m}=-x_{m-j, m}$ for $j=1,2, \ldots, m$. Given $m=1,2, \ldots$, a well-known inequality of Bernstein yields

$$
\frac{|Q(y)|}{\|Q\|_{\infty}} \leqslant \frac{\left|T_{m}(y)\right|}{\left\|T_{m}\right\|_{\infty}}=2^{m-1} \prod_{j=1}^{m}\left(y-x_{j, m}\right) \leqslant 2^{m}|y|^{m}, \quad y \in \mathbb{R} \backslash(-1,1),
$$

for every $Q \in \Pi_{m}$. Now let

$$
w(z)=|\omega| \prod_{j=1}^{k}\left|z-z_{j}\right|^{r_{j}} \in|\mathrm{GCAP}|_{\Gamma} .
$$

If each exponent $r_{j}$ is a positive rational number, say, $r_{j}=q_{j} / q$ for $j=1,2, \ldots, k$, with some positive integers $q_{j}$ and $q$, then applying (6.1) to the polynomial

$$
Q(z)=\omega^{2 q} \prod_{j=1}^{k}\left(z-z_{j}\right)^{q_{j}}\left(z-\bar{z}_{j}\right)^{q_{j}} \in \Pi_{2 q \Gamma}
$$

and taking the $2 q$ th root of its modulus, we obtain the inequality in the lemma. The case of positive real exponents $r_{j}$ in (6.2) can be reduced to that of rational exponents by approximation.

Proof of Theorem 2.1. Let $v$ denote the Chebyshev weight, that is, $v(x)=(1 / \pi)\left(1-x^{2}\right)^{-1 / 2}$, and let

$$
T_{m}(x)=\cos (m \theta) \quad \text { and } \quad U_{m}(x)=\frac{\sin ((m+1) 0)}{\sin \theta}, \quad-1 \leqslant x \leqslant 1, x=\cos \theta,
$$

be the Chebyshev polynomials of first and second kinds, respectively. We define the corresponding reproducing kernel function $K_{m}(v)$ by

$$
K_{m}(v, x, t)=1+2 \sum_{j=1}^{m} T_{j}(x) T_{j}(t)
$$

It is easy to see that

$$
K_{m}(v, x, x)=\frac{2 m+1+U_{2 m}(x)}{2},
$$


and, thus, by a straightforward calculation,

$$
c_{14}(m+1) \leqslant K_{m}(v, x, x)<2 m+1, \quad-1 \leqslant x \leqslant 1,
$$

where $c_{14}>0$ is an absolute constant. Therefore,

$$
\left|\frac{K_{m}(v, x, t)}{K_{m}(v, x, x)}\right| \leqslant \frac{2 m+1}{c_{14}(m+1)} \leqslant \frac{2}{c_{14}}, \quad-1 \leqslant x, t \leqslant 1 .
$$

In addition, we also have ${ }^{1}$

$$
\begin{array}{r}
\left|K_{m}(v, x, t)\right| \leqslant c_{15} \min \left\{m, 1+\frac{\sqrt{1-x^{2}}+\sqrt{2-t^{2}}}{|x-t|}\right\}, \\
-1 \leqslant x, t \leqslant 1, m=1,2, \ldots,
\end{array}
$$

with an absolute constant $c_{15}>0$. To show (6.6), we may assume without loss of generality that $0 \leqslant x \leqslant 1$, that is, $x=\cos \gamma$ with $0 \leqslant \gamma \leqslant \pi / 2$. By the Christoffel-Darboux formula,

$$
K_{m}(v, x, t)=\frac{T_{m}(x) T_{m-1}(t)-T_{m}(t) T_{m-1}(x)}{x-t} .
$$

First, by (6.3),

$$
\left|K_{m}(v, x, t)\right| \leqslant 2 m+1, \quad-1 \leqslant t \leqslant 1 .
$$

Second, if $t=\cos \theta$ with $0 \leqslant \theta \leqslant 2 \pi / 3$, then

$$
\begin{aligned}
& \left|T_{m}(x) T_{m-1}(t)-T_{m}(t) T_{m-1}(x)\right| \\
& \quad=\left|T_{m-1}(t)\left(T_{m}(x)-T_{m-1}(x)\right)-T_{m-1}(x)\left(T_{m}(t)-T_{m-1}(t)\right)\right| \\
& \quad \leqslant|\cos (m \gamma)-\cos ((m-1) \gamma)|+|\cos (m \theta)-\cos ((m-1) \theta)| \\
& \quad \leqslant 2|\sin ((2 m-1) \gamma / 2) \sin (\gamma / 2)|+2 \mid \sin ((2 m-1) \theta / 2) \sin (\theta / 2) ! \\
& \quad \leqslant\left|\frac{\sin \gamma}{\cos (\gamma / 2)}\right|+\left|\frac{\sin \theta}{\cos (\theta / 2)}\right| \leqslant 2\left(\sqrt{1-x^{2}}+\sqrt{1-\xi^{2}}\right)
\end{aligned}
$$

Thus, inequality (6.6) follows from (6.8) and (6.9).

Now, given $0<p<\infty, 0 \leqslant \Gamma<\infty$, and $n=1,2, \ldots$, let ${ }^{2}$

$$
s=\left[\frac{2 \Gamma+4}{p}\right]+1 \quad \text { and } \quad m=\left[\frac{n-1}{s}\right] .
$$

${ }^{1}$ See $[9$, Iemma 6.3 .8$, p. 108$]$ where the constant 1 was accidentally left out.

2 Here $[\cdot]$ denotes the integer part. 
Given $x \in[-1,1]$, define the polynomial $Q=Q_{x} \in \Pi_{n-1}$ by

$$
Q(t)=\left(\frac{K_{m}(v, x, t)}{K_{m}(v, x, x)}\right)^{s}
$$

If $m=0$, then $p(n-1) \leqslant 2 \Gamma+4$ and $Q(t) \equiv 1$, so we can obtain the inequality in Theorem 2.1 immediately as an application of (5.1) and Lemma 6.1. More specifically,

$$
\begin{aligned}
\lambda_{n}(\alpha, p, x) \leqslant & \int_{-1}^{1} w(t) d t \leqslant 2 \max _{-1 \leqslant t \leqslant 1} w(t) \\
\leqslant & 2\left(\frac{4}{\Delta_{M}(x)}\right)^{\Gamma} \max _{|t-x| \leqslant \Delta_{M}(x)} w(t) \\
\leqslant & 2\left(\frac{4}{\Delta_{M}(x)}\right)^{\Gamma} \Delta_{m}^{-1}(x) c_{8}^{2}(\Gamma+1)^{2} w_{M}(x) \\
\leqslant & \frac{1}{2}\left[2\left(1+\frac{p(n-1)}{\Gamma+p+1}\right)\right]^{2 \Gamma+2} c_{8}^{2}(\Gamma+1)^{2} w_{M}(x) \\
\leqslant & \frac{1}{2}\left[2\left(1+\frac{2 \Gamma+4}{\Gamma+p+1}\right)\right]^{2 \Gamma+2} c_{8}^{2}(\Gamma+1)^{2} \omega_{M}(x) \leqslant c_{16}^{\Gamma+1} w_{M}(x), \\
& \quad-1 \leqslant x \leqslant 1,
\end{aligned}
$$

where $c_{16}>0$ is an absolute constant.

Thus, in what follows, we may assume $m \geqslant 1$. We may also assume without loss of generality that $0 \leqslant x \leqslant 1$. For the sake of brevity, we introduce the intervals

$$
I_{m}(x)=\left[x-\Delta_{m}(x), x+\Delta_{m}(x)\right], \quad-1 \leqslant x \leqslant 1 .
$$

It follows from (6.5), (6.10), and (6.11) that the inequality

$$
\int_{I_{m}(x) \cap[-1,1]}|Q(t)|^{p} w(t) d t \leqslant c_{17}^{2 \Gamma+4+p} \Delta_{m}(x) \max _{t \in I_{m}(x)} w(t)
$$

holds with an absolute constant $c_{17}>0$. By Lemma 6.1,

$$
w(t) \leqslant\left[\frac{2|t-x|}{\Delta_{m}(x)}\right]^{\Gamma} \max _{y \in I_{m}(x)} w(y), \quad t \notin I_{m}(x),
$$

for every $w \in|\mathrm{GCAP}|_{\Gamma}$. 
Using (6.11), (6.10), (6.6), (6.4), and elementary estimates, it can be shown that the inequalities

$$
\begin{aligned}
& |Q(t)|^{p} \leqslant c_{18}^{2 \Gamma+4+p}\left(\frac{\sqrt{1-x^{2}}}{m|x-t|}\right)^{\Gamma+2}, \quad t \in[2 x-1,1] \backslash I_{m}(x), \\
& |Q(t)|^{p} \leqslant c_{18}^{5 \Gamma+4+p}\left(m \sqrt{1-t^{2}}\right)^{-2 \Gamma-4}, \quad t \in[-0.5,2 x-1] \backslash I_{m}(x), x \geqslant 0.5,
\end{aligned}
$$

and

$$
|Q(t)|^{p} \leqslant c_{18}^{2 \Gamma+4+p} m^{-2 \Gamma-4}, \quad x, t \in[-1,1],[t-x \mid \geqslant 0.5,
$$

hold with some absolute constant $c_{18}>0$, and we can assume that $c_{18} \geqslant 4$. For instance, to prove (6.14), we notice that $s p>\Gamma+2$, and $1+\left(\sqrt{1-x^{2}}+\sqrt{1-t^{2}}\right)|x-t|^{-1}$ on the right-hand side of (6.6) can be estimated by $5 \sqrt{1-x^{2}}|x-t|^{-1}$ for $t \in[2 x-1,1] \backslash I_{m}(x)$, whereas for (6.15), we use $1-x \leqslant 1-t$ and $0 \leqslant x-t \leqslant 1-t \leqslant 2(x-t)$ for $t \in[-0.5,2 x-1]$ and $x \geqslant 0.5$.

Using (6.13) and (6.14) we obtain

$$
\begin{aligned}
& \int_{[2 x-1.1] \backslash I_{m}(x)}|Q(t)|^{p} w(t) d t \\
& \leqslant 2^{\Gamma} c_{18}^{2 \Gamma+4+p}\left(\frac{\sqrt{1-x^{2}}}{m \Delta_{m}(x)}\right)^{\Gamma} \Delta_{m}^{2}(x) \\
& \times \max _{y \in I_{m}(x)} w(y) \int_{[2 x-1,1] \backslash I_{m}(x)}|t-x|^{-2} d t \\
& \leqslant c_{18}^{3 \Gamma+5+p} \Delta_{m}(x) \max _{y \in I_{m}(x)} w(y) .
\end{aligned}
$$

In addition, by (6.13), (6.15), and by $0 \leqslant x-t \leqslant 1-t$,

$$
\begin{aligned}
& \int_{[-0.5 .2 . x-1] \backslash \Lambda_{m}(x)}|Q(t)|^{p} w(t) d t \\
& \leqslant 2^{T} c_{18}^{2 \Gamma+4+p} m^{-2 \Gamma-4} \max _{y \in I_{m}(x)} w(y) \\
& \times \int_{[-0.5,2 x-1], I_{m}(x)}\left[\frac{\left(1-t^{2}\right)}{\Delta_{m}(x)}\right]^{\Gamma}\left(1-t^{2}\right)^{-\Gamma-2} d t \\
& \leqslant c_{18}^{3 \Gamma+4+p^{-4}} \max _{y \in I_{m}(x)} w(y) \int_{L-0.5,2 x-1 \jmath ; I_{m}(x)}\left(1-t^{2}\right)^{-2} d t \\
& \leqslant c_{18}^{3 \Gamma+5+p} m^{-4} \Delta_{m}^{-1}(x) \max _{y \in I_{m}(x)} w(y) \leqslant c_{18}^{3 \Gamma^{+5+p}} \Delta_{m}(x) \max _{y \in I_{m}(x)} w(y)
\end{aligned}
$$


for $0.5 \leqslant x \leqslant 1$. Finally, from (6.13) and (6.16) we obtain

$$
\begin{aligned}
& \int_{[-1,1] \backslash[x-0.5, x+0.5]}|Q(t)|^{p} w(t) d t \\
& \quad \leqslant 4^{T} c_{18}^{2 \Gamma+4+p} m^{-4}\left(m^{2} \Delta_{m}(x)\right)^{-\Gamma} \max _{y \in I_{m}(x)} w(y) \\
& \leqslant c_{18}^{3 \Gamma+4+p} \Delta_{m}(x) \max _{y \in I_{m}(x)} w(y)
\end{aligned}
$$

by a generous estimate, where we used the fact that $|x-t| \leqslant 2$ in (6.13). Since $Q \in \Pi_{n-1}$ and $Q(x)=1$, the extremal property (1.5) and the inequalities (6.12) and (6.17)-(6.19) yield

$$
\lambda_{n}(\alpha, p, x) \leqslant \int_{-1}^{1}|Q(t)|^{p} w(t) d t \leqslant c_{19}^{r+p+1} \Delta_{m}(x) \max _{y \in I_{m}(x)} w(y)
$$

with an absolute constant $c_{19} \geqslant 4$. By $(6.10)$,

$$
\frac{p(n-1)}{2 \Gamma+4+p} \leqslant m \leqslant \frac{p(n-1)}{2 \Gamma+4} .
$$

Therefore, Lemma 6.1, inequalities (6.21), the facts that $m \geqslant 1$ and $M=1+p(n-1) /(\Gamma+p+1)$, and inequality (5.1) imply

$$
\Delta_{m}(x) \max _{y \in I_{m}(x)} w(y) \leqslant c_{20}^{\Gamma+p+1} \Delta_{M}(x) \max _{y \in I_{M}(x)} w(y) \leqslant c_{21}^{\Gamma^{+}}+p+1 w_{M}(x)
$$

with some appropriately chosen absolute constants $c_{20}>0$ and $c_{21}>0$, which together with (6.20) yields Theorem 2.1.

Proof of Theorem 2.2. We will prove the appropriate upper bound for $\lambda_{n}$ only; the corresponding estimate for $\lambda_{n}^{*}$ is analogous, and it requires only minor modifications of the following proof. Let

$$
w^{(B)}(z)=|\omega| \prod_{j=1}^{d}\left|z-z_{j}\right|^{r_{j}} \in|\mathrm{GCAP}|_{\Gamma}, \quad z_{i} \neq z_{j} \quad \text { if } \quad i \neq j
$$

Set $^{3}$

$$
\begin{gathered}
q_{j}=\left[\frac{r_{j}}{p}\right]+1, \quad q=\sum_{j=1}^{k} q_{j} \leqslant \frac{\Gamma+p d}{p}, \\
s=\left[\frac{2 \Gamma+4}{p}\right]+1, \quad m=\left[\frac{n-1-q}{s}\right] \geqslant 1, \\
P(\cdot)=|\omega| \prod_{j=1}^{d}\left(\cdot-z_{j}\right)^{q_{j} \in \Pi_{q},}
\end{gathered}
$$

${ }^{3}$ Here $[\cdot]$ denotes the integer part. 
and

$$
\widetilde{P}_{x}(\cdot)=|\omega| \prod_{j=1}^{d}\left(\cdot-\tilde{z}_{j}\right)^{y_{j}} \in \Pi_{q}, \quad-1 \leqslant x \leqslant 1,
$$

where

$$
\tilde{z}_{j}= \begin{cases}z_{j}, & \text { if } \quad\left|z_{j}-x\right| \geqslant \frac{1}{2} \Delta_{m}(x) \\ x-\Delta_{m}(x), & \text { if } \quad\left|z_{j}-x\right|<\frac{1}{2} A_{m}(x)\end{cases}
$$

Let

$$
Q(\cdot)=\left(\frac{K_{m}(v, x, \cdot)}{K_{m}(v, x, x)}\right)^{s} \tilde{P}_{x}(\cdot) \in \Pi_{n-1} .
$$

Obviously,

$$
|Q(x)|^{p}=\left|\widetilde{P}_{x}(x)\right|^{p}
$$

Introducing the sets

$$
J_{1}=\left[x-\Delta_{m}(x), x+A_{m}(x)\right] \cap[-1,1] \quad \text { and } \quad J_{2}=[-1,1] \backslash J_{i},
$$

replacing $w$ by $W=\left|\widetilde{P}_{x}\right|^{p} w \in|\mathrm{GCAP}|_{\Gamma+p d}$ in the proof of Theorem 2.1, and using (6.22)-(6.30) and (5.1), we obtain

$$
\begin{aligned}
\int_{J_{2}}|Q(t)|^{p} w(t) d t & =\int_{J_{2}}\left|\frac{K_{m}(v, x, t)}{K_{m}(v, x, x)}\right|^{s p}\left|\tilde{P}_{x}(t)\right|^{p} w(t) d t \\
& \leqslant 4^{\Gamma+p d} \int_{J_{2}}\left|\frac{K_{m}(v, x, t)}{K_{m}(v, x, x)}\right|^{s p}|P(t)|^{p} w(t) d t \\
& \leqslant 4^{\Gamma+p d} c_{22}^{\Gamma+p d+p+1} \int_{|t-x| \leqslant \Delta_{m}(x)}|P(t)|^{p} w(t) d t
\end{aligned}
$$

with an absolute constant $c_{22}>0$ chosen in such a way that $c_{19}^{\Gamma+p d+p+1} c_{8}^{2}(\Gamma+p d+1)^{2} \leqslant c_{22}^{\Gamma+p d+p+1}$. Here we used that $\left|\widetilde{P}_{x}(t)\right| \leqslant$ $4^{\Gamma+p d}|P(t)|$ for every $t \in J_{2}$ which follows from (6.22)-(6.27). Observe that (6.22)-(6.27) and (6.30) imply $\left\{\left.P(t)\right|^{p} \leqslant 2^{\Gamma+p d}\left|\tilde{P}_{. x}(x)\right|^{p}\right.$ for every $t \in J_{2}$. This, together with (6.31), yields

$$
\begin{aligned}
\int_{J_{2}}|Q(t)|^{p} w(t) d t & \leqslant 8^{\Gamma+p d} c_{22}^{\Gamma+p d+p+1}\left|\widetilde{P}_{x}(x)\right|^{p} \int_{|t-x| \leqslant \Delta_{m}(x)} w(t) d t \\
& \leqslant c_{23}^{\Gamma+p d+p+1}\left|\widetilde{P}_{x}(x)\right|^{p} \int_{|l-x| \leqslant \Delta_{m}(x)} w(t) d t
\end{aligned}
$$

with an absolute constant $c_{23}>0$. In addition, (6.22)-(6.27) and (6.30) 
imply $\left|\widetilde{P}_{x}(t)\right|^{p} \leqslant 3^{r+p d}\left|\tilde{P}_{x}(x)\right|^{p}$ for every $t \in J_{1}$, so that by (6.28), (6.23), $(6.24)$, and (6.5)

$$
\begin{aligned}
\int_{J_{1}}|Q(t)|^{p} w(t) d t & \leqslant c_{24}^{2 \Gamma+4+p} \int_{J_{1}}\left|\widetilde{P}_{x}(t)\right|^{p} w(t) d t \\
& \leqslant c_{24}^{2 \Gamma+4+p} 3^{\Gamma+p d}\left|\widetilde{P}_{x}(x)\right|^{p} \int_{J_{1}} w(t) d t \\
& \leqslant c_{25}^{\Gamma+p d+p+1}\left|\widetilde{P}_{x}(x)\right|^{p} \int_{J_{1}} w(t) d t
\end{aligned}
$$

where $c_{24}>0$ and $c_{25}>0$ are absolute constants. Therefore, the extremal property (1.5) and formulas (6.32), (6.33), (6.30), (6.28), (6.23), and (6.24) yield

$$
\begin{aligned}
\lambda_{n}(\alpha, p, x) & \leqslant\left|\widetilde{P}_{x}(x)\right|^{-p} \int_{-1}^{1}|Q(t)|^{p} w(t) d t \leqslant c_{26}^{\Gamma^{+}+p d+p+1} w_{m}(x) \\
& \leqslant c_{26}^{\Gamma^{+p d+p+p+1} w_{M}(x)}
\end{aligned}
$$

where $c_{26}>0$ is an absolute constant and $M=(p(n-1-d)-\Gamma) /(2 \Gamma+4+p)$, and, hence, the theorem has completely been proved.

\section{ProOf of Theorems 3.1 aNd 3.2}

Proof of Theorem 3.1. Let $x \in[-1,1]$ be fixed, $N=\Gamma+p(n-1)$, and $M=1+p(n-1) /(\Gamma+p+1)$, and $\operatorname{let}^{4}$

$$
I_{M, \eta}(x)=\left[x-\eta \Delta_{M}(x), x+\eta \Delta_{M}(x)\right], \quad 0<\eta \leqslant 1 .
$$

Let the weight $w \in|\mathrm{GCAP}|_{\Gamma}$ be of the form

$$
w(z)=|\omega| \prod_{j=1}^{k}\left|z-z_{j}\right|^{r_{j}}, \quad \omega \neq 0, z_{j} \in \mathbb{C}, z \in \mathbb{C}, r_{j}>0, \sum_{j=1}^{k} r_{j} \leqslant \Gamma .
$$

In what follows, $A_{M}(x)$ denotes the open disk in the complex plane centered at $x$ with radius $0.125 \cdot \Delta_{M}(x)$. Define $\tilde{z}_{j}$ and $\tilde{w}$ by

$$
\tilde{z}_{j}= \begin{cases}z_{j}, & \text { if } z_{j} \in \mathbb{C} \backslash A_{M}(x) \\ x-0.125 \cdot \operatorname{sgn}(x) \Delta_{M}(x), & \text { if } z_{j} \in A_{M}(x),\end{cases}
$$

and

$$
\tilde{w}(\cdot)=|\omega| \prod_{j=1}^{k}\left|\cdot-\tilde{z}_{j}\right|^{r_{j}} \in|\mathrm{GCAP}|_{\Gamma}
$$

${ }^{4}$ See formula (1.7) for the definition of $\Delta_{M}(x)$. 
respectively. In what follows, we assume that $Q \in|\mathrm{GCAP}|_{n-1}$. Observe that $Q \in|\mathrm{GCAP}|_{n-1}$ and $\tilde{w} \in|\mathrm{GCAP}|_{\Gamma}$ imply $|Q|^{p} \tilde{w} \in|\mathrm{GCAP}|_{N}$ with $N=\Gamma+p(n-1)$, and, therefore, using Theorem 5.1 with $0<s=$ $\Delta_{M}(x)<1$, where $M=1+p(n-1) /(\Gamma+p+1)$, we obtain

$$
\begin{aligned}
& m\left(\left\{t \in[-1,1]:|Q(t)|^{p} \hat{w}(t) \geqslant \exp \left(-c_{7}(I+p+1)\right)|Q(x)|^{p} \tilde{w}(x)\right\}\right) \\
& \geqslant \Delta_{M}(x) .
\end{aligned}
$$

Hence, there is a set

$$
E \subset[-1,1] \backslash\left[x-0.25 \cdot \Delta_{M}(x), x+0.25 \cdot A_{M}(x)\right]
$$

such that

$$
A_{M}(x) \leqslant 2 m(E)
$$

and

$$
|Q(t)|^{p} \tilde{w}(t) \geqslant \exp \left(-c_{7}(\Gamma+p+1)\right)|Q(x)|^{p} \tilde{w}(x), \quad t \in E .
$$

Observe that (7.1), (7.2), (7.3), and (7.4) yield

$$
\tilde{w}(x) \geqslant(2 / 3)^{\Gamma} \max _{i \in I_{m, 0.0625}(x)} w(t)
$$

and

$$
w(t) \geqslant 3^{-\Gamma} \tilde{w}(t), \quad t \in E
$$

From inequalities (7.5), (7.6), and (7.7) we obtain

$$
\begin{aligned}
& \frac{2 \exp \left(c_{7}(\Gamma+p+1)\right)}{\Delta_{M}(x)} \int_{E}|Q(t)|^{p} \tilde{w}(t) d t \\
& \quad \geqslant|Q(x)|^{p} \tilde{w}(x) \geqslant|Q(x)|^{p}(2 / 3)^{\Gamma} \max _{t \in I_{I_{L} .0025(x)}} w(t) .
\end{aligned}
$$

We can use this estimate combined with (7.8) and Lemma 6.1 to obtain the existence of an absolute constant $c_{27}>0$ such that

$$
\begin{aligned}
\int_{-1}^{1}|Q(t)|^{p} w(t) d t \\
\quad \geqslant \int_{E}|Q(t)|^{p} w(t) d t \geqslant 3^{-\Gamma} \int_{E}|Q(t)|^{p} \tilde{w}(t) d t \\
\quad \geqslant 3^{-\Gamma} \exp \left(-c_{7}(\Gamma+1)\right) 2^{-1} \Delta_{M}(x)(2 / 3)^{I}|Q(x)|^{p} \max _{t \in I_{M, 0062 S(x)}} w(t) \\
\quad \geqslant c_{27}^{\Gamma^{+p+1}} \Delta_{M}(x)|Q(x)|^{p} \max _{|t-x| \leqslant \Delta_{M}(x)} w(t) \geqslant c_{27}^{\Gamma^{+p+1}}|Q(x)|^{p} w_{M}(x)
\end{aligned}
$$


Since (7.9) holds for every $Q \in \mid$ GCAP $\left.\right|_{n-1}$, Theorem 3.1 has completely been proved in view of the extremal property (1.6) of the generalized Christoffel function $\lambda^{*}$.

Proof of Theorem 3.2. In what follows we will assume that $0<p<\infty$ and $x \in[-1,1]$. We start with the inequality

$$
\begin{gathered}
\left|P_{n}(z)\right|^{p}|g(r z)|^{2} \leqslant \frac{(2+p n) e}{8 \pi} \int_{-\pi}^{\pi}\left|P_{n}\left(e^{i \theta}\right)\right|^{p}\left|g\left(e^{i \theta}\right)\right|^{2} d \theta, \\
|z|=1, \quad r=\frac{p n}{p n+2},
\end{gathered}
$$

which holds for every $g \in H^{2}(|z| \leqslant 1)$ and for every complex algebraic polynomial $P_{n}$ of degree at most $n=1,2, \ldots$ (cf. [7, Theorem 6, p. 148]). A simple calculation shows that for every real trigonometric polynomial $R_{n}$ of degree at most $n$ there is an algebraic polynomial $P_{2 n} \in \Pi_{2 n}$ such that $R_{n}^{2}(\theta)=\left|P_{2 n}\left(e^{i \theta}\right)\right|^{2}$. Therefore, (7.10) yiclds

$$
\begin{gathered}
\left|R_{n}(\gamma)\right|^{p}\left|g\left(r e^{i \gamma}\right)\right|^{2} \leqslant \frac{(1+p n) e}{4 \pi} \int_{-\pi}^{\pi}\left|R_{n}(\theta)\right|^{p}\left|g\left(e^{i \theta}\right)\right|^{2} d \theta, \\
\gamma \in \mathbb{R}, \quad r=\frac{p n}{p n+2},
\end{gathered}
$$

holds for every real trigonometric polynomial $R_{n}$ and for every $g \in H^{2}(|z| \leqslant 1)$. First, we extend (7.11) to every $f \in|\mathrm{GCTP}|_{N}$, writing $N$ in place of $n$. To this end, first assume $f$ can be written as

$$
f(z)=|\omega| \prod_{j=1}^{k}\left|\sin \frac{z-z_{j}}{2}\right|^{r_{j}}, \quad \omega \neq 0, z_{j} \in \mathbb{C}, z \in \mathbb{C}, \sum_{j=1}^{k} r_{j} \leqslant 2 N,
$$

with some rational exponents $r_{j}>0$. If $r_{j}=q_{j} / q$ with some positive integers $q_{j}$ and $q$ for $1 \leqslant j \leqslant k$, then, applying (7.11) with $p /(2 q)$ instead of $p$ to the trigonometric polynomial $R_{n}$ of degree at most $2 q N$, where

$$
R_{n}(z)=|\omega|^{2 q} \prod_{j=1}^{k}\left(\sin \frac{z-z_{j}}{2} \sin \frac{z-\bar{z}_{j}}{2}\right)^{q_{j}}
$$

we obtain

$$
\begin{gathered}
|f(\gamma)|^{p}\left|g\left(r e^{i \gamma}\right)\right|^{2} \leqslant \frac{(1+p N) e}{4 \pi} \int_{-\pi}^{\pi}|f(\theta)|^{p}\left|g\left(e^{i \theta}\right)\right|^{2} d \theta, \\
\gamma \in \mathbb{R}, \quad r=\frac{p N}{p N+2},
\end{gathered}
$$


for every $f \in|\mathrm{GCTP}|_{N}$ and for every $g \in H^{2}(|z| \leqslant 1)$. Now let $1 \leqslant n<\infty$, $0<p<\infty$, and let $Q \in|\mathrm{GCAP}|_{n-1}$. Applying (7.12) with $f$, where $f(\theta)=$ $|Q(\cos \theta)||\sin \theta|^{1, p} \in|\mathrm{GCTP}|_{n-1+1 / p}$, and choosing $g=D(w(\cos \cdot)) \in$ $H^{2}(|z| \leqslant 1)$, we obtain

$$
\begin{gathered}
\left.\left|Q\left(\cos \gamma^{1}\right)\right|^{p} \mid \sin \gamma\right)\left.|| D\left(w(\cos \theta), r e^{i \gamma}\right)\right|^{2} \\
\leqslant \frac{(2+p(n-1)) e}{4 \pi} \int_{-\pi}^{\pi}\left|Q_{n}(\cos \theta)\right|^{p}|\sin \theta|\left|D\left(w(\cos \cdot), e^{i \theta}\right)\right|^{2} d \theta \\
\quad r=\frac{p(n-1)+1}{p(n-1)+3},
\end{gathered}
$$

for every $Q \in|\mathrm{GCAP}|_{n-1}$. Since $\left|D\left(w(\cos \cdot), e^{i \dagger}\right)\right|^{2}=w(\cos \theta)$ for almost every real $\theta$, we can use the change of variables $t=\cos \theta$ and $x=\cos \gamma$ to obtain from (7.13) the inequality

$$
\begin{aligned}
& |Q(x)|^{p} \sqrt{1-x^{2}} \\
& \leqslant\left|D\left(w(\cos \cdot), r e^{i \theta}\right)\right|^{-2} \frac{(2+p(n-1)) 2 e}{4 \pi} \int_{-1}^{1}|Q(t)|^{p} w(t) d t, \\
& r=\frac{p(n-1)+1}{p(n-1)+3},
\end{aligned}
$$

for every $Q \in|\operatorname{GCAP}|_{n-1}$. To finish the proof, we refer to our Schur-type inequality [5, Theorem 7] which states that given $0<N<\infty$ and $0<p<\infty$,

$$
\max _{-1 \leqslant t \leqslant 1}|Q(t)|^{p} \leqslant e(1+p N) \max _{-1 \leqslant t \leqslant 1}\left(\sqrt{1-t^{2}}|Q(t)|^{p}\right)
$$

for every $Q \in|\mathrm{GCAP}|_{N}$. Now Theorem 3.2 follows from the last two inequalities, and from the definition of $\Delta_{M}(x)$ in $(1.7)$.

\section{Proof of Theorem 4.1}

Proof of Theorem 4.1. Given $n=1,2, \ldots$ and $0 \leqslant \Gamma<\infty$, let $M=1+2(n-1) /(\Gamma+3)$. First we prove (4.1). It is sufficient to prove the existence of a positive constant $c_{28}$ such that

$x_{m-1, n}-x_{m, n} \leqslant c_{28}^{\Gamma+1}\left(\Delta_{M}\left(x_{m-1, n}\right)+\Delta_{M}\left(x_{m, n}\right)\right), \quad m=1,2, \ldots, n+1$,

since (8.1) implies (4.1) by a straightforward calculation (cf. (1.7)). Assume that there is an integer $m$ such that $1 \leqslant m \leqslant n+1$ and

$$
x_{m-1, n}-x_{m, n}>A_{M}\left(x_{m-1, n}\right)+A_{M}\left(x_{m . n}\right)
$$


since, otherwise, we already have the desired upper bound for this particular value of $m$. We introduce the Christoffel numbers

$$
\lambda_{m, n}=\lambda_{n}\left(\alpha, 2, x_{m . n}\right), \quad m=0,1, \ldots, n+1 .
$$

By the Markov-Stieltjes inequality [6, formula (5.4), p. 29] we have

$$
\int_{x_{m, n}}^{x_{m-1, n}} w(t) d t \leqslant \lambda_{m-1, n}+\lambda_{m, n}, \quad m=1,2, \ldots, n+1
$$

Since $w \in|\operatorname{GCAP}|_{\Gamma}$, Theorem 2.1 yields

$$
\begin{aligned}
\lambda_{m, n} & \leqslant c_{1}^{\Gamma+3} w_{M}\left(x_{m, n}\right)=c_{1}^{\Gamma+3} \int_{\left|t-x_{m, n}\right| \leqslant \Delta_{M}\left(x_{m, n}\right)} w(t) d t \\
& \leqslant c_{1}^{\Gamma+3} \Delta_{M}\left(x_{m, n}\right) \max _{\left|t-x_{m, n}\right| \leqslant \Delta_{M}\left(x_{m, n}\right)} w(t), \quad m=0,1, \ldots, n+1 .
\end{aligned}
$$

Observe that assumption (8.2) and Lemma 6.1 imply

$$
\max _{\left|t-x_{m, n}\right| \leqslant \Delta_{M}\left(x_{m, n}\right)} w(t) \leqslant 6^{\Gamma} \max _{x_{m, n} \leqslant t \leqslant x_{m-1 . n}} w(t), \quad m=1,2, \ldots, n+1,
$$

and

$$
\max _{\left|t-x_{m-1, n \mid}\right| \leqslant \Delta_{m}\left(x_{m-1, n}\right)} w(t) \leqslant 6^{r} \max _{x_{m, n} \leqslant t \leqslant x_{m-1, n}} w(t), \quad m=1,2, \ldots, n+1 .
$$

Now inequalities (8.4)-(8.7) and Theorem 5.2 yield

$$
\begin{aligned}
& \left(x_{m-1, n}-x_{m, n}\right) \max _{x_{m, n} \leqslant t \leqslant x_{m-1, n}} w(t) \\
& \quad \leqslant 2 c_{8}^{2}(\Gamma+1)^{2} \int_{x_{m, n}}^{x_{m-1, n}} w(t) d t \leqslant 2 c_{8}^{2}(\Gamma+1)^{2}\left(\lambda_{m-1, n}+\lambda_{m, n}\right) \\
& \quad \leqslant 2 c_{8}^{2}(\Gamma+1)^{2} c_{1}^{\Gamma+3} 6^{\Gamma}\left(\Delta_{M}\left(x_{m-1, n}\right)+\Delta_{M}\left(x_{m, n}\right)\right) \max _{x_{m, n} \leqslant t \leqslant x_{m-1, n}} w(t),
\end{aligned}
$$

for $m=1,2, \ldots, n+1$, and, thus, (8.1) follows. Hence, (4.1) has been proved.

The proof of (4.2) is somewhat more complicated; it is based on a method of Erdös and Turán (cf. [6, pp. 111-112; 8, p. 369; 9, pp. 164-165]). Given $n=1,2, \ldots$, and $0 \leqslant \Gamma<\infty$, let $M=1+2(n-1) /(\Gamma+3)$, and let

$$
I_{M, \eta}(x)=\left[x-\eta \Delta_{M}(x), x+\eta \Delta_{M}(x)\right], \quad 0<\eta \leqslant 1 .
$$


Let $\left\{l_{m, n}\right\}_{m=1}^{n}$ denote the fundamental polynomials of Lagrange interpolation defined by

$$
l_{m, n} \in \Pi_{n-1} \quad \text { and } \quad l_{m, n}\left(x_{j, n}\right)=\delta_{j, m}, j, m=1,2, \ldots, n,
$$

where $\delta_{j, m}$ is the Kronecker-delta symbol. The identity

$$
\frac{1}{\lambda_{n}(\alpha, 2, t)}=\sum_{j=1}^{n} \frac{l_{j . n}^{2}(t)}{\lambda_{j, n}}
$$

where $\lambda_{j, n}$ is given by (8.3), is well known (cf. [6, formula (4.7), p. 25]). From (8.9), Theorems 2.1 and 3.1, Lemma 6.1, and (5.1) we obtain

$$
\begin{aligned}
l_{m, n}^{2}(t) & =\frac{l_{m, n}^{2}(t)}{\lambda_{m, n}} \lambda_{m, n} \leqslant \sum_{j=1}^{n} \frac{l_{j, n}^{2}(t)}{\lambda_{j, n}} \lambda_{m, n}=\frac{\lambda_{m, n}}{\lambda_{n}(\alpha, 2, t)} \\
& \leqslant c_{29}^{\Gamma+3} \frac{\Delta_{M}\left(x_{m, n}\right) \max _{\left|y-x_{m, n}\right| \leqslant \Delta M\left(x_{m, n}\right)} w(y)}{\Delta_{M}(t) \max _{|y-t| \leqslant A_{M}(t)} w(y)} \leqslant c_{30}^{r+1}, t \in I_{m, 1} \cap[-1,1]
\end{aligned}
$$

with some appropriate absolute constants $c_{29}>0$ and $c_{30}>0$. Let $A_{n 2}(x)$ denote the open disk centered at $x$ with radius $0.5 \cdot A_{n}(x)$. Given

$$
w(\cdot)=\prod_{j=1}^{k}\left|\cdot-z_{j}\right|^{r^{\prime},}, \quad \sum_{j=1}^{k} r_{j} \leqslant \Gamma
$$

we define

$$
\tilde{z}_{j}= \begin{cases}z_{j}, & \text { if } \quad z_{j} \in \mathbb{C} \backslash A_{n}\left(x_{m, n}\right\} \\ x_{m, n}-0.5 \cdot \operatorname{sgn}\left(x_{m, n}\right) \Delta_{M}\left(x_{m, n}\right), & \text { if } \quad z_{j} \in A_{n}\left(x_{m, n}\right)\end{cases}
$$

and

$$
\tilde{w}(\cdot)=\prod_{j=1}^{k}\left|\cdot-\tilde{z}_{j}\right|^{r^{\prime}}
$$

We will estimate

$$
\int_{-1}^{1} l_{m, n}^{2}(t) \tilde{w}(t) d t
$$

To this end we introduce the sets

$$
J_{1}=I_{M, 1}\left(x_{m, n}\right) \cap[-1,1] \quad \text { and } \quad J_{2}=[-1,1] \backslash J_{1} .
$$


It follows from (8.10)

$$
\begin{aligned}
\int_{J_{1}} l_{m, n}^{2}(t) \tilde{w}(t) d t & \leqslant c_{30}^{\Gamma+1} \Delta_{M}\left(x_{m, n}\right) \max _{\left|t-x_{m, n}\right| \leqslant \Delta_{M}\left(x_{m, n}\right)} \tilde{w}(t) \\
& \leqslant c_{30}^{\Gamma+1} \Delta_{M}\left(x_{m, n}\right) \cdot 8^{\Gamma} \max _{\left|t-x_{m, n}\right| \leqslant 0.25 \cdot \Delta_{M}\left(x_{m . n}\right)} \tilde{w}(t) .
\end{aligned}
$$

Observe that (8.11) -(8.14) imply $\tilde{w}(t) \leqslant 3^{\Gamma} w(t)$ for $t \in J_{2}$ and $w(t) \leqslant 3^{\Gamma} \tilde{w}(t)$ for $t \in I_{M, 0.25}\left(x_{m . n}\right)$. Therefore, by the Gauss-Jacobi quadrature formula (cf. [6, formula (3.9), p.23]), Theorem 2.1 and Lemma 6.1 yield

$$
\begin{aligned}
& \int_{J_{2}} l_{m, n}^{2}(t) \tilde{w}(t) d t \\
& \quad \leqslant 3^{\Gamma} \int_{J_{2}} l_{m, n}^{2}(t) w(t) d t \leqslant 3^{\Gamma} \int_{-1}^{1} l_{m, n}^{2}(t) w(t) d t=3^{\Gamma} \lambda_{m, n} \\
& \quad=3^{\Gamma} \lambda_{n}\left(\alpha, 2, x_{m, n}\right) \leqslant 3^{\Gamma} c_{1}^{\Gamma+3} \Delta_{M}\left(x_{m, n}\right) \max _{\left|t-x_{m, n}\right| \leqslant \Delta_{M}\left(x_{m, n}\right)} w(t) \\
& \quad \leqslant 3^{\Gamma} c_{1}^{\Gamma+3} \Delta_{M}\left(x_{m, n}\right) \cdot 8^{\Gamma} \max _{\left|z-x_{m, n}\right| \leqslant 0.25 \cdot \Delta_{M}\left(x_{m, n}\right)} w(t) \\
& \quad \leqslant 3^{\Gamma} c_{1}^{\Gamma+3} \Delta_{M}\left(x_{m, n}\right) \cdot 8^{\Gamma} \cdot 3^{\Gamma} \max _{\left|t-x_{m, n}\right| \leqslant 0.25 \cdot \Delta_{M}\left(x_{m, n}\right)} \tilde{w}(t) .
\end{aligned}
$$

Summarizing (8.15) and (8.16), we get

$$
\int_{-1}^{1} l_{m, n}^{2}(t) \tilde{w}(t) d t \leqslant c_{31}^{\Gamma+1} \Delta_{M}\left(x_{m, n}\right) \max _{\left|t-x_{m, n}\right| \leqslant 0.25 \cdot \Delta_{M}\left(x_{m, n}\right)} \tilde{w}(t)
$$

with an appropriate absolute constant $c_{31}>0$. When proving (4.2), we can assume without loss of generality that $x_{m, n} \leqslant 0$. We may also assume that $x_{m-1, n} \leqslant 0.25$; otherwise, there is nothing to prove. Now Theorem 5.4, (8.17), and the inequalities $x_{m, n} \leqslant 0$ and $x_{m-1, n} \leqslant 0.25$ imply

$$
\begin{aligned}
l_{m, n}^{2}(x) \tilde{w}(x) & \leqslant c_{10}\left(\Delta_{2 n+\Gamma}(x)\right)^{-1} \int_{-1}^{1} l_{m, n}^{2}(t) \tilde{w}(t) d t \\
& \leqslant c_{32}^{\Gamma+1}\left(\Delta_{2 n+\Gamma}(x)\right)^{-1} \Delta_{M}\left(x_{m, n}\right) \max _{\left|t-x_{m, n}\right| \leqslant 0.25 \cdot \Delta_{M}\left(x_{m, n}\right)} \tilde{w}(t) \\
& \leqslant c_{33}^{\Gamma+1} \max _{\left|t-x_{m, n}\right| \leqslant 0.25 \cdot \Delta_{M}\left(x_{m, n}\right)} \tilde{w}(t) \\
& |x| \leqslant\left|x_{m, n}\right|+0.5 \cdot\left(1-\left|x_{m, n}\right|\right)
\end{aligned}
$$


with appropriate absolute constants $c_{32}>0$ and $c_{33}>0$. Assume that

$$
x_{m-1 . n}-x_{m, n} \leqslant 0.25 \cdot \Delta_{M}\left(x_{m . n}\right),
$$

since, otherwise, we already have the desired lower bound for this particular value of $m$. In view of (8.11)-(8.13) and (8.19), we have

$$
\tilde{w}\left(x_{m, n}\right) \leqslant(1.5)^{\Gamma} \tilde{w}(\xi), \quad \forall \xi \in\left[x_{m, n}, x_{m-1 . n}\right],
$$

and

$$
\max _{\left|t-x_{m, n}\right| \leqslant 0.25 \cdot \Delta_{M}\left(x_{m, n}\right)} \tilde{w}(t) \leqslant(1.5)^{\Gamma} \tilde{w}\left(x_{m, n}\right)
$$

Finally, using (8.8), the Mean Value Theorem, inequality (5.2) transformed linearly to the interval

$$
[-y, y] \equiv\left[-\left|x_{m, n}\right|-0.5 \cdot\left(1-\left|x_{m, n}\right|\right),\left|x_{m, n}\right|+0.5 \cdot\left(1-\left|x_{m, n}\right|\right)\right],
$$

the assumptions $x_{m, n} \leqslant 0$ and $x_{m-1, n} \leqslant 0.25$, and inequalities (8.18), (8.20), and (8.21), we can find a point $\xi \in\left(x_{m . n}, x_{m-1, n}\right)$ such that

$$
\begin{aligned}
\tilde{w}\left(x_{m, n}\right) & =\left|l_{m, n}^{2}\left(x_{m-1, n}\right)-l_{m, n}^{2}\left(x_{m, n}\right)\right| \tilde{w}\left(x_{m, n}\right) \\
& =\left(x_{m-1, n}-x_{m, n}\right)\left|\left(l_{m, n}^{2}\right)^{\prime}(\xi) \tilde{w}\left(x_{m, n}\right)\right| \\
& \leqslant\left(x_{m-1, n}-x_{m, n}\right)(1.5)^{\Gamma}\left|\left(l_{m, n}^{2}\right)^{\prime}(\xi) \tilde{w}(\xi)\right| \\
& \leqslant\left(x_{m-1, n}-x_{m, n}\right) c_{34}(\Gamma+1)^{2} y^{-1} \Delta_{n}^{-1}(\xi / y) \max _{|x| \leqslant\left|x_{m, n}\right|+0.5 \cdot\left(1-\left|x_{m, n}\right|\right)} \tilde{w}(x) \\
& \leqslant\left(x_{m-1, n}-x_{m, n}\right) c_{35}^{\Gamma+1} A_{M}^{-1}\left(x_{m, n}\right) c_{16}^{\Gamma+1} \max _{\left|r-x_{m, n}\right| \leqslant 0.25 \cdot \Delta M\left(x_{m, n}\right)} \tilde{w}(t) \\
& \leqslant\left(x_{m-1, n}-x_{m, n}\right) c_{36}^{\Gamma+1} \Delta_{M}^{-1}\left(x_{m, n}\right)(1.5)^{I} \tilde{w}\left(x_{m, n}\right)
\end{aligned}
$$

with appropriate absolute constants $c_{34}>0, c_{35}>0$, and $c_{36}>0$, and this, together with (1.7), gives the lower bound in (4.2). Thus, the theorem has completely been proved.

\section{REFERENCES}

1. T. ERDÉLYI, Remez-type inequalities on the size of generalized polynomials, J. London Math. Soc., to appear.

2. T. ERdÉLYI, Markov and Bernstein type inequalities for generalized non-negative polynomials, Canad. J. Math. 43 (1991), 495-505.

3. T. FRDíryr, Nikol'skiñ-type inequalities for generalized polynomials and zeros of orthogonal polynomials, J. Approx. Theory 66 (1991), $80-92$. 
4. T. ERDÉLYI, Weighted Markov and Bernstein type inequalities for generalized non-negative polynomials, J. Approx. Theory 68 (1992), 283-305.

5. T. ERdélyi, A. MÁté, AND P. Neval, Inequalities for generalized non-negative polynomials, Constr. Approx., to appear.

6. G. Freud, "Orthogonal Polynomials," Pergamon, Oxford, 1971.

7. A. MÁtÉ AND P. NevaI, Berstein's inequality in $L^{p}$ for $0<p<1$ and $(C, 1)$ bounds for orthogonal polynomials, Ann. of Math. 111 (1980), 145-154.

8. P. NevaI, Mean convergence of Lagrange interpolation, I, J. Approx. Theory 18 (1976), 363-377.

9. P. Neval, "Orthogonal Polynomials," Mem. Amer. Math. Soc., Vol. 213, Amer. Math. Soc., Providence, RI, 1979. 